\title{
Beyond renoprotective impact; ameliorative effects of metformin on malignancies
}

\author{
Nozar Dorestan ${ }^{1}$, Mohammad Bahadoram ${ }^{2}$, Mohammadreza Khosravi ${ }^{3}$, Sara Bahadoram ${ }^{4}$, Mohammad \\ Davoodi $^{5}$, Maedeh Barahman ${ }^{6 *}$ \\ ${ }^{1}$ Department of Surgery, School of Medicine, Ahvaz Jundishapur University of Medical Sciences, Ahvaz, Iran \\ ${ }^{2}$ Medical Student Research Committee and Social Determinant of Health Research Center, Ahvaz Jundishapur University of Medical Sciences, \\ Ahvaz, Iran \\ ${ }^{3}$ Cancer Prevention Research Center, Isfahan University of Medical Sciences, Isfahan, Iran \\ ${ }^{4}$ Department of Pediatrics, Imam Khomeini Hospital Complex, Tehran University of Medical Sciences, Tehran, Iran \\ ${ }^{5}$ Department of Radiology, School of Medicine, Ahvaz Jundishapur University of Medical Sciences, Ahvaz, Iran \\ ${ }^{6}$ Department of Radiation Oncology, Firoozgar Hospital, Firoozgar Clinical Research Development Center (FCRDC), Iran University of Medical \\ Sciences (IUMS), Tehran, Iran
}

\section{A R T I C L E IN F O}

\section{Article Type:}

Review

\section{Article History:}

Received: 14 November 2017

Accepted: 20 February 2018

Published online: 3 March 2018

\section{Keywords:}

Metformin

Prostate cancer

Liver cancer

Breast cancer

Lung cancer

Colon cancer

\begin{abstract}
A B S T R A C T
Diabetes therapies were associated with either a reduced or increased risk of cancer. There are continuous discussions about a possible protective action of metformin. In fact, metformin, a commonly consumed anti-diabetic medication has an ameliorative impact on some cancers by direct (preventing cell growth and apoptosis) and indirect (increasing insulin sensitivity) effects. Observational reports have suggested which metformin reduces the incidence of few common cancers. In general, metformin is an extensively recommended anti-diabetic medication with a recognized ability coupled with a satisfactory and safety profile and inexpensive. This review article considers reduction and protective abilities of metformin on cancers especially prostate, liver, breast, lung, and colon cancer.
\end{abstract}

Implication for health policy/practice/research/medical education:

Metformin, a commonly consumed anti-diabetic medication, has an ameliorative impact on some cancers by direct (preventing cell growth and apoptosis) and indirect (increasing insulin sensitivity) effects.

Please cite this paper as: Dorestan N, Bahadoram M, Khosravi M, Bahadoram S, Davoodi M, Barahman M. Beyond renoprotective impact; ameliorative effects of metformin on malignancies. J Nephropharmacol. 2017;7(2):50-54.

\section{Introduction}

1,1-dimethylbiguanide hydrochloride has known metformin, marketed under the trade name 'Glucophage' among others, is an oral glucose-lowering drug. In fact, in the early 20th century it was mentioned to lower blood glucose concentrations in animals, however it was not until the 1950s that Jean Sterne examined metformin and subsequently developed 'Glucophage' (1). In other words, metformin is the most recommended oral drug with antihyperglycemic properties and is intended the first choice for treatment recently identified type II diabetes by several expert diabetes institutes (2).

In addition, with nearly 50 years of collected worldwide medical experience, metformin is mostly considered as safe with the most common side effects being gastrointestinal in nature such as nausea, diarrhea, and to the lowest amount, vomiting. In the same way, in comparison with most other drugs consumed to treat T2DM, metformin does not produce weight gain, and is commonly related to a low-risk of low blood sugar (2).

On the other hand, reports have exhibited a link between increased cancer risk and diabetes in addition to adverse 
effects in cancer persons (3).

In addition, metformin raises adenosine monophosphateactivated protein kinase (AMPK), reduces gluconeogenesis, and manages insulin levels. Several reports have shown the risk of cancer suffering in diabetes patients who used metformin is lower (10\%-50\%) than non-consumer. Therefore, it is hypothesized that metformin has protective effects against cancer. Many studies have been detected the mechanisms of reaction against cancer cell that prevent cancer cell migration and advance apoptosis (4). In addition, many observational examinations also examined the effects of metformin not as prevention, but as a therapy for cancer, by examining persons identified with cancer of colorectal, prostate, ovarian, breast and lung cancer (5).

The objectives of this paper are to review the studies conducted protective effects of metformin on cancers especially liver, prostate, breast, lung, and colon cancers.

\section{Materials and Methods}

For this review, we used a variety of sources including PubMed, Embase, EBSCO, Scopus and directory of open access journals (DOAJ). The search was conducted by using combinations of the following key words and/or their equivalents; metformin, prostate cancer, liver cancer, breast cancer, lung cancer, renoprotection and colon cancer.

\section{Effects of metformin on liver cancer}

Liver cancer is the 10th most common cancer and the fifth most common cause of cancer death among men. It is also the eighth most common cause of cancer death among women. The incidence of this cancer in men is almost three times that of women. In 2017 the incidence of this cancer has tripled since 1980. Hepatic malignancy is much more common in Southeast Asia and sub-Saharan Africa than in the United States. Liver cancer is the most usual cancer in some countries (1).

Hepatocellular carcinoma (HCC) is the maximum kind of initial liver cancer. Despite the fact that the prevalence of some cancers is decreasing, the prevalence of HCC are growing global (6). Additionally, HCC is usually related to a poor diagnosis. Most patients are identified when the disease is progressive. As a result, recognition and chemopreventive treatments of persons at risk for HCC indicate one of the most excellent approaches for decreasing death due to this cancer (7).

Various risks cause liver cancer contain hepatitis $\mathrm{C}$ virus (HCV) infection, hepatitis $\mathrm{B}$ virus infection, and over intake of alcohol and non-alcoholic fatty liver, which, nevertheless, cannot describe the rapid rise of liver cancer in certain areas (7). On the other hand, epidemiologic data shows a rising part for diabetes in the growth of HCC. Respective risk of HCC is two to three times more in patients with type II diabetes (7).

Meanwhile, metformin controls basal glucose production by controlling glycogenolysis and gluconeogenesis in the liver and rising glucose uptake in the muscle (8). Many studies have assessed the effects of metformin on liver cancer $(7,8)$. In general, it shows to be related to a less risk of liver cancer in persons with type II diabetes (8).

In 2012, Zhi-Jiang et al reviewed evidence accessible to evaluate chemoprevention effect of metformin for liver cancer in type II diabetes patients (8). Their results show that metformin is related to an estimated decrease of $62 \%$ in the risk of liver cancer among patients with type II diabetes. When the analysis is limited to four reports that examined HCC, metformin is related to an estimated $70 \%$ decrease in the risk of this cancer (8).

Generally these studies present which metformin avoids HCC and protective effects of metformin for HCC should be evaluated in at-risk subjects (9). In spite of this findings, more examination, counting well-designed cohort investigations, mechanistic investigations, and possibly controlled examinations, are necessary.

\section{Effects of metformin on prostate cancer}

Prostate cancer is the most commonly diagnosed male cancer (2) and second directing cause of cancer mortality among men in the Western population (9). Even though the main reason for growth of prostatic carcinoma is complex, a new agreement report from the American Diabetes Association (ADA) and the American Cancer Society (ACS) stressed on a relation between prostate cancer and diabetes mellitus (10).

Indeed, hyperinsulinemia has harmful side effects and may increase the risk of death by prostatic carcinoma (10). Likewise, recent findings have detected a comparative risk decrease in the occurrence of prostate cancer in persons using metformin (4). Actually, the role of metformin in prostatic cancer is under evaluation, while one large experimental investigation displayed no decrease of risk (10), one more investigation showed $44 \%$ decrease of risk in prostate cancer incidence (11). To recognize etiologies of prostate cancer, four experimental investigations evaluating the effect of metformin. Two of the investigations showing advanced survival, one of them showing nonsignificant reduced chances of high-grade tumor and cancer progress, and another result showed no effect of metformin on later removal of prostate gland (4). In other words, these results have led to rising attention in pharmacologically aiming pathways of metabolism, particularly pathways of metabolism included in the enhancement of insulin resistance. Since metformin is an oral hypoglycemic agent, it reverses hyperinsulinemia in persons with and without diabetes, and it can be considered as an effective treatment drug for prostate cancer (10). Besides of this findings, metformin has been detected to prevent the rapid growth of prostate cancer cell sequences. In vitro proposed mechanism for metformin that causes stopping of abnormal growth of tissue (antineoplastic effects) is preventing the mechanistic target of rapamycin 
(mTOR) signaling transduction downstream through activation AMPK pathway (10). AMPK is a target for cancer treatment because of its effect on cell growth. Generally, diabetic persons with prostatic carcinoma who consumed metformin have a better recovery in prostatic carcinoma.

\section{Effects of metformin on breast cancer}

Most prevalent invasive cancer in females is breast cancer that causes cancer mortality in female after lung carcinoma (12). It can arise in both women and men, but it's very common in women (13).

In fact, both type 2 diabetes and obesity have been related to increased breast cancer by hyperinsulinemia as a possible mediating factor (14). Thus metformin may have a favorable influence on breast cancer progress (3).

Many epidemiological and clinical studies have demonstrated that the insulin resistance, hyperinsulinemia, and obesity may be the main risk for certain kinds of cancers, such as breast cancer. Therefore, treatment with metformin may ameliorate progress of some malignancies. It has been shown that metformin has inhibitory impact on cancer cells via direct (effect on cell growth and death) or indirect (effect on insulin sensitivity) (15). A pilot work has shown a positive effect of metformin in breast cancer (16). In addition, long-time administration of metformin was related to a decreased possibility of rising breast cancer, although no such effect was observed with shorttime administration (3).

In another investigation, among 68019 postmenopausal females that were examined in long-time of 11.8 years, 3273 occurrences of invasive breast cancer were identified (16). Diabetic females taking metformin had lower breast cancer incidence, while females taking other diabetic drugs had a slightly upper incidence of breast cancer in contrast with non-diabetic females (17).

The sensitivity to insulin and rising blood glucose levels decrease by metformin administration via preventing of gluconeogenesis in liver and increase glucose uptake in skeletal muscle. Even though numerous mechanisms of metformin actions have been suggested, activation of AMPK is most important action and leads to fatty acid and glucose oxidation and avoid from progress of cancer (16). Similarly, AMPK is a main cellular energy sensor. Activation of AMPK leads to inhibition of many of the procedures highly reliant on sufficient cellular adenosine triphosphate source, containing protein, gluconeogenesis and cholesterol biosynthesis and, fatty acid synthesis, as well as raise of catabolic procedures such as glycolysis and fatty acid beta-oxidation (16). These findings are strong preclinical rationales for a potential positive effect of metformin on breast cancer.

\section{Effects of metformin on lung cancer}

Lung cancer (both small cell and non-small cell) is the second most common cancer in both men and women (not counting skin cancer). In men, prostate cancer is more common, while in women breast cancer is more common. About $14 \%$ of all new cancers are lung cancers. Lung cancer is by far the leading cause of cancer death among both men and women. Each year, more people die of lung cancer than of colon, breast, and prostate cancers combined. Lung cancer often occurs in older people. The average age at the time of lung cancer diagnosis is about 70 years. Most people diagnosed with lung cancer are 65 or older, while few patients have age below the age of 45 at diagnosis) $(3,18)$.

During the last few years, few reports on the association of lung cancer with metformin have been published. These reports are evidence from some epidemiological reports, in-vitro and in-vivo studies and mechanistic examinations (17).

However, some other studies detected metformin prevents lung carcinoma development and causes cell death of lung carcinoma (19). It was detected that metformin inhibited lung tumorigenesis made by 4-(methylnitrosamino)-1(3-pyridyl)-1-butanone (NNK), a tobacco cancer (19). Likewise, many epidemiologic examinations, but not all, have exhibited a particularly lower chance of lung cancer related to metformin administration. Accordingly, metformin treatment was related to assessed decreases of $29 \%$ in lung carcinoma and $15 \%$ in cancers of the respiratory system. The contradictory results of these studies were smoking status and cancer site (19).

As a result, metformin therapy shows to be connected with a considerably lower chance of lung carcinoma in diabetic persons.

\section{Effects of metformin on colon cancer}

Colorectal cancer (CRC) is the third most commonly diagnosed malignancy throughout the world and the fourth leading cause of tumor mortality. The survival of CRC depends on the stage of the disease at the time of diagnosis. Useful factors for the prognosis of individuals with CRC comprise early diagnosis and timely and appropriate treatment, while harmful factors known for prognosis is heavy alcohol consumption, obesity and diabetes mellitus. Diabetics have a higher risk of CRC incidence and mortality than nondiabetics. Regarding the relationship between metformin and CRC, some investigations did not find any relation between administration of metformin and the overall survival of CRC in patients while other investigations showed a protective role regarding decreasing the CRC-specific and overall mortality, as well as prolonging the overall survival. According to new studies in diabetic patients, the association between the administration of metformin and prognosis of CRC is still controversial (4,20-22).

\section{Discussion}

Metformin is a drug with a good safety outline that has been consumed since the 1950s as the first choice for 
treatment of type II diabetes and that is presently being studied as a cancer therapy (5). In addition, earlier epidemiological examinations have shown a link between long-time administration of metformin among diabetic persons and lesser risks of cancer and cancer death in general (8).

On the other hand, the major analysis, based on five observational examinations, shows that metformin is related with a $31 \%$ statistically noteworthy decrease in cancer chance compared with other diabetic therapies, even though with some degrees of heterogeneity among reports (26). Importantly, several preclinical surveys verify this pleiotropic effect of metformin on cancer inhibition. Metformin prevents the proliferation of pancreatic cancer cells (19), endometrial cancer (23), ovarian cancer (24), and breast cancer (15). As well as metformin prevents tumor progress in animal models of lung cancer, colon cancer, and breast cancer (8). These results are in agreement with the primary epidemiological results. Moreover, metformin has been exhibited to inhibit carcinogenesis due to direct and indirect impacts. Furthermore, hyperinsulinemia enhances cancer chance in healthy persons and can partially describe a relation between the obesity and cancer risk in many body organs, concluding kidney, colon, endometrial, breast, gastrointestinal and pancreatic cancers $(25,26)$. As well, high glucose levels is a risk agent for numerous cancers, such as breast and colon (26). In spite of this finding regarding high blood glucose and hyperinsulinemia, however, a direct anticancer influence for metformin have existed, while preclinical findings have shown that metformin can prevent the growth of cancer cells in vitro and in vivo (26). However, the exact antitumor impact of metformin remained to be elucidated.

Furthermore, metformin increases insulin sensitivity in peripheral tissues and so improves endogenous hyperinsulinemia (8) that in turn can theoretically link to a lower chance of liver cancer (8). One more possible explanation related to the consumption of metformin might be the activation of AMPK that prevents the m-TOR pathway, a significant positive manager of cell proliferation and translation initiation. Additional possible procedures essential the antitumor effect of metformin might be anti-inflammation (25), weight loss (26), or decreasing endogenous reactive oxygen species (27).

Accordingly, main advantages of metformin contain which it is administered orally, economical, and has a very satisfactory toxicological portrait. Lactic acidosis is the most adverse effect of metformin administration that is very rare (10). In addition, metformin is harmless in non-diabetic persons and accepted for disorders except diabetes such as polycystic ovary syndrome and renoprotection (10).

Metformin is a threshold of a novel production of metformin-based medicines with purpose of cancer treatment (28).

\section{Conclusion}

This review highlighted that more studies particularly intended for assessment of metformin and cancer are required.

\section{Authors' contribution}

$\mathrm{MoB}$ and SB: Idea and writing the manuscript. $\mathrm{MK}$ and MB: Literature Review. MD and ND: critique and thought. All authors read and approved the final manuscript.

\section{Conflicts of interest}

The authors declare no conflict of interest.

\section{Ethical considerations}

Ethical issues (including plagiarism, data fabrication, double publication) have been completely observed by the authors.

\section{Funding/Support}

This study was funded by Vice Chancellor of Research Affairs of Ahvaz Jundishapur University of Medical Sciences.

\section{References}

1. Bailey CJ, Day C. Metformin: its botanical background. Pract Diabetes Int. 2004;21:115-7. doi: 10.1002/pdi.606.

2. DeFronzo R, Fleming GA, Chen K, Bicsak TA. Metforminassociated lactic acidosis: Current perspectives on causes and risk. Metabolism. 2016;65:20-9. doi: 10.1016/j. metabol.2015.10.014

3. Rizos CV, Elisaf MS. Metformin and cancer. Eur J Pharmacol. 2013;705:96-108.doi:10.1016/j.ejphar.2013.02.038.

4. Kaushik D, Karnes RJ, Eisenberg MS, Rangel LJ, Carlson RE, Bergstralh EJ. Effect of metformin on prostate cancer outcomes after radical prostatectomy. Urol Oncol. 2014;32: 43.e1-43.e7. doi: 10.1016/j.urolonc.2013.05.005.

5. Suissa S. Metformin to Treat Cancer: Misstep in Translational Research from Observational Studies. Epidemiology. 2017;28:455-8. doi: 10.1097/EDE.0000000000000634.

6. Bhalla K, Hwang BJ, Dewi RE, Twaddel W, Goloubeva OG, Wong KK, et al. Metformin prevents liver tumorigenesis by inhibiting pathways driving hepatic lipogenesis. Cancer Prev Res. 2012;5:544-52. doi: 10.1158/1940-6207.CAPR11-0228.

7. Li X, Wang X, Gao P. Diabetes Mellitus and Risk of Hepatocellular Carcinoma. Biomed Res Int. 2017;2017:5202684. doi: $\quad 10.1155 / 2017 / 5202684$

8. Tajima K, Nakamura A, Shirakawa J, Togashi Y, Orime $\mathrm{K}$, Sato K, et al. Metformin prevents liver tumorigenesis induced by high-fat diet in C57Bl/6 mice. Am J Physiol Endocrinol Metab. 2013;305:E987-E98. doi: 10.1152/ ajpendo.00133.2013.

9. Spratt DE, Zhang C, Zumsteg ZS, Pei X, Zhang Z, Zelefsky MJ. Metformin and prostate cancer: reduced development of castration-resistant disease and prostate cancer mortality. Eur Urol. 2013;63:709-16. doi: 10.1016/j. eururo.2012.12.004.

10. Currie CJ, Poole CD, Gale EA. The influence of glucoselowering therapies on cancer risk in type 2 diabetes. Diabetologia. 2009;52:1766-77. doi: 10.1007/s00125-009- 
1440-6.

11. Wright JL, Stanford JL. Metformin use and prostate cancer in Caucasian men: results from a population-based casecontrol study. Cancer Causes Control. 2009;20:1617-22. doi: 10.1007/s10552-009-9407-y.

12. Christian N, Christina C. What you need to know about breast cancer 2017. Available from: https://www. medicalnewstoday.com/articles/37136.php.

13. Mayo Clinic Staff. Breast cancer 2017. Available from: https://www.mayoclinic.org/diseases-conditions/breastcancer/symptoms-causes/syc-20352470? $\mathrm{p}=1$.

14. Col NF, Ochs L, Springmann V, Aragaki AK, Chlebowski RT. Metformin and breast cancer risk: a meta-analysis and critical literature review. Breast Cancer Res Treat. 2012;135:639-46. doi: 10.1007/s10549-012-2170-x.

15. Goodwin PJ, Ligibel JA, Stambolic V. Metformin in breast cancer: time for action. J Clin Oncol. 2009. 27:3271-3. doi: 10.1200/JCO.2009.22.1630.

16. Chlebowski RT, McTiernan A, Wactawski-Wende J, Manson JE, Aragaki AK, Rohan T, et al. Diabetes, metformin, and breast cancer in postmenopausal women. J Clin Oncol. 2012;30:2844-52. doi: 10.1200/JCO.2011.39.7505.

17. Yendamuri S. Metformin and lung cancer. J Clin Sci Res. 2014;3:26-32.

18. Liver Cancer: Statistics. Cancer.Net website. http://www. cancer.net/cancer-types/liver-cancer/statistics. Accessed 15 February 152017.

19. Kisfalvi K, Eibl G, Sinnett-Smith J, Rozengurt E. Metformin disrupts crosstalk between $G$ protein-coupled receptor and insulin receptor signaling systems and inhibits pancreatic cancer growth. Cancer Res. 2009;69:6539-45. doi: 10.1158/0008-5472.CAN-09-0418.

20. Whitburn J, Edwards CM, Sooriakumaran P. Metformin and Prostate Cancer: a New Role for an Old Drug. Curr
Urol Rep. 2017;18:46. doi: 10.1007/s11934-017-0693-8.

21. Non-small cell lung cancer. American Cancer Society; 2017. Available from: https://www.cancer.org/cancer/nonsmall-cell-lung-cancer.html. Accessed August 29, 2017

22. Meng F, Song L, Wang W. Metformin Improves Overall Survival of Colorectal Cancer Patients with Diabetes: A Meta-Analysis. J Diabetes Res. 2017;2017:5063239. doi: $10.1155 / 2017 / 5063239$.

23. Tan BK, Adya R, Chen J, Lehnert H, Sant Cassia LJ, Randeva HS. Metformin treatment exerts antiinvasive and antimetastatic effects in human endometrial carcinoma cells. J Clin Endocrinol Metab. 2011;96:808-16. doi: 10.1210/jc.2010-1803.

24. Rattan R, Giri S, Hartmann LC, Shridhar V. Metformin attenuates ovarian cancer cell growth in an AMP-kinase dispensable manner. J Cell Mol Med. 2011;15:166-78. doi: 10.1111/j.1582-4934.2009.00954.x.

25. Grenader T, Goldberg A, Shavit L. Metformin as an addition to conventional chemotherapy in breast cancer. J Clin Oncol. 2009;27:e259-e. doi: 10.1200/JCO.2009.25.4110.

26. Krakoff J, Clark JM, Crandall JP, Wilson C, Molitch ME, Brancati FL, et al. Effects of metformin and weight loss on serum alanine aminotransferase activity in the diabetes prevention program. Obesity. 2010;18:1762-7. doi: 10.1038/ oby.2010.21.

27. Algire C, Moiseeva O, Deschênes-Simard X, Amrein L, Petruccelli L, Birman E, et al. Metformin reduces endogenous reactive oxygen species and associated DNA damage. Cancer Prev Res. 2012;5:536-43. doi: 10.1158/19406207.CAPR-11-0536.

28. Menendez JA, Martin-Castillo B, Joven J. Metformin and cancer: Quo vadis et cui bono? Oncotarget. 2016;7:54096101. doi: 10.18632/oncotarget.10262.

Copyright $\odot 2018$ The Author(s); Published by Society of Diabetic Nephropathy Prevention. This is an open-access article distributed under the terms of the Creative Commons Attribution License (http://creativecommons.org/licenses/by/4.0), which permits unrestricted use, distribution, and reproduction in any medium, provided the original work is properly cited. 